\title{
A NOTE ON THE GENERALIZED RIEMANN INTEGRAL
}

WASHEK F. PFEFFER

(Communicated by R. Daniel Mauldin)

Dedicated to Professor Ralph Henstock for his 65 th birthday

\begin{abstract}
We show that the generalized Riemann integral can be defined by means of gage functions which are upper semicontinuous when restricted to a suitable subset whose complement has measure zero.
\end{abstract}

By introducing $\delta$-fine partitions for a positive function $\delta$ (see below), Henstock and Kurzweil obtained a strikingly simple Riemannian definition of the DenjoyPeron integral (cf. Definition 1 and [S, Chapter VIII]). In their definition, the function $\delta$ is completely arbitrary, and it is not clear how complicated it need be (a question of P. S. Bullen-see [Q]). The purpose of this note is to establish that $\delta$ can be always selected so that it is upper semicontinuous when restricted to a suitable subset whose complement has measure zero (cf. [ $\mathbf{P}_{\mathbf{2}}$, Lemma 3]). The proof is quite simple: we show first that such a $\delta$ can be chosen if the integrand is Lebesgue integrable, and then we follow the constructive Denjoy definition, observing that the upper semicontinuity property of $\delta$ is preserved at the inductive step. We also show that for a bounded Lebesgue integrable function, a gage $\delta$ can be selected so that it is upper semicontinuous everywhere (cf. [FM, Example 1]).

The author is obliged to J. Foran for pointing out a serious error in the preprint of this paper.

By $\mathbf{R}$ and $\mathbf{R}_{+}$we denote the set of all real and all positive real numbers, respectively. Unless stated otherwise, all functions in this paper are real-valued. When no confusion is possible, we denote by the same symbol a function on a set $E$, as well as its restrictions to various subsets of $E$. An interval is a compact nondegenerate subinterval of $\mathbf{R}$. A collection of intervals whose interiors are disjoint is called a nonoverlapping collection. If $E \subset \mathbf{R}$, then $\mathrm{cl}(E)$, $\operatorname{int}(E), d(E)$, and $|E|$ denote, respectively, the closure, interior, diameter, and outer Lebesgue measure of $E$. A function $\delta$ on an interval $A$ is called nearly upper semicontinuous if there is a set $H \subset A$ such that $|A-H|=0$ and $\delta\lceil H$ is upper semicontinuous.

A subpartition of an interval $A$ is a collection $P=\left\{\left(A_{1}, x_{1}\right), \ldots,\left(A_{p}, x_{p}\right)\right\}$ where $A_{1}, \ldots, A_{p}$ are nonoverlapping subintervals of $A$, and $x_{i} \in A_{i}, i=1, \ldots, p$. If, in addition, $\bigcup_{i=1}^{p} A_{i}=A$, we say that $P$ is a partition of $A$. Given a $\delta: A \rightarrow \mathbf{R}_{+}$, we say that a subpartition $P$ is $\delta$-fine whenever $d\left(A_{i}\right)<\delta\left(x_{i}\right)$ for $i=1, \ldots, p$. An easy compactness argument shows that a $\delta$-fine partition of an interval $A$ exists for each $\delta: A \rightarrow \mathbf{R}_{+}$.

Received by the editors June 11, 1987.

1980 Mathematics Subject (Classification (1985 Revision). Primary 26A39.

(C) 1988 American Mathematical Society $0002-9939 / 88 \$ 1.00+\$ .25$ per page 
If $f$ is a function on an interval $A$ and $P=\left\{\left(A_{1}, x_{1}\right), \ldots,\left(A_{p}, x_{p}\right)\right\}$ is a subpartition of $A$, we let

$$
\sigma(f, P)=\sum_{i=1}^{p} f\left(x_{i}\right)\left|A_{i}\right| .
$$

1. Definition (HENSTOCK-KURzWEIL). A function $f$ on an interval $A$ is called integrable in $A$ if there is a real number $I$ with the following propety: given $\varepsilon>0$, we can find a $\delta: A \rightarrow \mathbf{R}_{+}$such that $|\sigma(f, P)-I|<\varepsilon$ for each $\delta$-fine partition $P$ of $A$.

Since $\delta$-fine partitions of an interval $A$ exist for each $\delta: A \rightarrow \mathbf{R}_{+}$, it is easy to see that the number $I$ from the previous definition is determined uniquely by the integrable function $f$. It is called the integral of $f$ over $A$, denoted by $\int_{A} f$, or $\int_{a}^{b} f$ if $A=[a, b]$. The family of all integrable functions on $A$ is denoted by $\mathscr{R}(A)$.

A detailed study of the integral defined above can be found in $[\mathbf{H}$ and $\mathbf{K}]$; an elementary exposition is given in $\left[\mathbf{M l}\right.$ and $\left.\mathbf{P}_{\mathbf{1}}\right]$. In particular, it is shown in $[\mathbf{K}$, Theorem 4.14 and $\mathbf{P}_{\mathbf{1}}$, Corollary B5] that the integral coincides with the DenjoyPerron integral (see [S, Chapter VIII, Theorems (3.9) and (3.11)]). As this result is important for our purposes, we formulate it precisely.

The family of all Denjoy-Perron integrable functions on an interval $A$ is denoted by $\mathscr{D}(A)$, and if $f \in \mathscr{D}(A)$, the symbol $(D) \int_{A} f$ denotes the Denjoy-Perron integral of $f$ over $A$.

2. THEOREM (HENSTOCK-KURZWEIL). If $A$ is an interval, then $\mathscr{R}(A)=$ $\mathscr{D}(A)$ and $\int_{A} f=(D) \int_{A} f$ for each $f \in \mathscr{R}(A)$.

The function $\delta$ from Definition 1 is often referred to as a gage associated to $f$ and $\varepsilon$. For an integrable function $f$ on an interval $A$ and an $\varepsilon>0$, we denote by $\Delta(f, A ; \varepsilon)$ the family of all gage functions associated to $f$ and $\varepsilon$. Since positive continuous functions on compact intervals are bounded away from zero, we see immediately that $f$ is Riemann integrable in the classical sense if and only if $\Delta(f, A ; \varepsilon)$ contains a continuous gage for each $\varepsilon>0$. Our goal is to show that for each $\varepsilon>0$, the family $\Delta(f, A ; \varepsilon)$ always contains a nearly upper semicontinuous gage. To this end, we denote by $\mathscr{R}_{*}(A)$ the family of all $f \in \mathscr{R}(A)$ such that $\Delta(f, A ; \varepsilon)$ contains a nearly upper semicontinuous function for each $\varepsilon>0$, and we show that $\mathscr{R}_{*}(A)=\mathscr{R}(A)$.

3. LEMMA. Let $h$ be a lower semicontinuous function on a set $E \subset \mathbf{R}$, let $\eta>0$, and for each $x \in E$, let $\delta(x)$ be the supremum of all numbers $\delta \in(0,1]$ such that $y \in E$ and $|y-x|<\delta$ implies $h(y) \geq h(x)-\eta$. Then the function $x \mapsto \delta(x)$ is upper semicontinuous on $E$.

PROOF. Proceeding towards a contradiction, suppose that there is an $x \in E$ and a sequence $\left\{x_{n}\right\}$ in $E$ such that $\lim x_{n}=x$ and $\lim \delta\left(x_{n}\right)>\delta(x)+\alpha$ for some $\alpha>0$. By the definition of $\delta(x)$, there is a $y \in E$ with $|y-x|<\delta(x)+\alpha / 2$ and $h(y)<h(x)-\eta$. Choose a $\beta>0$ so that $h(y)<h(x)-\eta-\beta$, and find an $x_{n}$ for which $\left|x-x_{n}\right|<\alpha / 2, \delta\left(x_{n}\right)>\delta(x)+\alpha$, and $h\left(x_{n}\right) \geq h(x)-\beta$. Then

$$
\left|y-x_{n}\right| \leq|y-x|+\left|x-x_{n}\right|<\delta(x)+\alpha<\delta\left(x_{n}\right) \text {, }
$$

and hence $h(y) \geq h\left(x_{n}\right)-\eta \geq h(x)-\eta-\beta$; a contradiction.

If $E$ is a Lebesgue measurable subset of $\mathbf{R}$, we denote by $\mathscr{L}(E)$ the family of all functions $f$ on $E$ for which the finite Lebesgue integral $(L) \int_{E} f$ exists. 
4. Proposition. If $A$ is an interval, then $\mathscr{L}(A) \subset \mathscr{R}_{*}(A)$.

ProOF. Let $f \in \mathscr{L}(A), \varepsilon>0$, and let $\eta=\varepsilon /(|A|+1)$. There is an uper semicontinuous function $g: A \rightarrow[-\infty,+\infty)$, and a lower semicontinuous function $h: A \rightarrow(-\infty,+\infty]$ such that $g \leq f \leq h$ and $(L) \int_{A}(h-g)<\eta$. Let $E=\{x \in$ $A: h(x)<+\infty\}$, and let $\delta_{h}$ be the positive upper semicontinuous function on $E$ associated to $h \uparrow E$ and $\eta$ according to Lemma 3. If $x \in A-E$, we select any $\delta_{h}(x)>0$ so that $h(y) \geq f(x)-\eta$ for each $y \in A$ with $|y-x|<\delta_{h}(x)$. Since $|A-E|=0$, we have defined a nearly upper semicontinuous function $\delta_{h}: A \rightarrow \mathbf{R}_{+}$. Using $-g$ instead of $h$, we define similarly a nearly upper semicontinuous function $\delta_{g}: A \rightarrow \mathbf{R}_{+}$, and set $\delta=\min \left(\delta_{h}, \delta_{g}\right)$. Now if $P=\left\{\left(A_{1}, x_{1}\right), \ldots,\left(A_{p}, x_{p}\right)\right\}$ is a $\delta$-fine partition of $A$, then $g(x) \leq f\left(x_{i}\right)+\eta$ and $h(x) \geq f\left(x_{i}\right)-\eta$ for each $x \in A_{i}$, $i=1, \ldots, p$. Thus $(L) \int_{A_{i}} g \leq(L) \int_{A_{i}} f \leq(L) \int_{A_{i}} h$, and

$$
\text { (L) } \int_{A_{i}} g-\eta\left|A_{i}\right| \leq f\left(x_{i}\right)\left|A_{i}\right| \leq(L) \int_{A_{i}} h+\eta\left|A_{i}\right|, \quad i=1, \ldots, p .
$$

It follows that

$$
\begin{aligned}
\left|\sigma(f, P)-(L) \int_{A} f\right| & \leq \sum_{i=1}^{p}\left|f\left(x_{i}\right)\right| A_{i}\left|-(L) \int_{A_{i}} f\right| \\
& \leq \sum_{i=1}^{p}\left[\eta\left|A_{i}\right|+(L) \int_{A_{i}}(h-g)\right]<\varepsilon
\end{aligned}
$$

and we have $f \in \mathscr{R}_{*}(A)$.

If $f \in \mathscr{L}(A)$ then, in general, $\Delta(f, A ; \varepsilon)$ may contain no gage which is a Baire functions on the whole interval $A$ (see [FM, Example 1]). However, a closer look at the proof of Proposition 4 shows that $\Delta(f, A ; \varepsilon)$ contains an upper semicontinuous gage for each $\varepsilon>0$ whenever $f$ has an upper semicontinuous majorant and a lower semicontinuous minorant which are both finite. In particular, we have the following corollary.

5. COROLLARY. If $f$ is a bounded Lebesgue integrable function on an interval $A$, then $\Delta(f, A ; \varepsilon)$ contains an upper semicontinuous gage for every $\varepsilon>0$.

6. REMARK. The proofs of Proposition 4 and Corollary 5 translate verbatim to the higher dimensional Henstock-Kurzweil integrals, as well as to the integral defined by $\mathrm{McSh}$ ane in [Ms]. Since the McShane integral coincides with that of Lebesgue (see $\left[\mathbf{P}_{\mathbf{1}}\right.$, Corollary $\mathrm{B} 11$ or $\left.\mathbf{M l}, \S 8.3\right]$ ), we see that it can be always defined by means of nearly upper semicontinuous gages, which can be taken upper semicontinuous whenever the integrand is bounded. This remains true even for a general setting discussed in $[\mathbf{A P}]$, provided the underlying space is metrizable.

7. LEMMA. Let $A=[a, b]$ be an interval.

(i) The family $\mathscr{R}_{*}(A)$ is a real vector space.

(ii) If $f \in \mathscr{R}_{*}(A)$, then $f \in \mathscr{R}_{*}(B)$ for each subinterval $B$ of $A$.

(iii) If $f \in \mathscr{R}_{*}(A)$ and $\varepsilon>0$, then there is a nearly upper semicontinuous function $\delta: A \rightarrow \mathbf{R}_{+}$such that

$$
\sum_{i=1}^{p}\left|f\left(x_{i}\right)\right| A_{i}\left|-\int_{A_{i}} f\right|<\varepsilon
$$

for each $\delta$-fine subpartition $\left\{\left(A_{1}, x_{1}\right), \ldots,\left(A_{p}, x_{p}\right)\right\}$ of $A$. 
(iv) If $c \in(a, b)$ and $f: A \rightarrow \mathbf{R}$ belongs to $\mathscr{R}_{*}([a, c])$ and $\mathscr{R}_{*}([c, b])$, then $f$ belongs also to $\mathscr{R}_{*}([a, b])$.

(v) If $f: A \rightarrow \mathbf{R}$ belongs to $\mathscr{R}_{*}([a, c])$ for each $c \in(a, b)$, and a finite limit $\lim _{c \rightarrow b-} \int_{a}^{c} f=I$ exists, then $f \in \mathscr{R}_{*}([a, b])$ and $\int_{a}^{b} f=I$.

PROOF. The proofs of properties (i) $-(\mathrm{v})$ are the same as those of the corresponding properties of the Henstock-Kurzweil integral (cf. [Ml, §§2.1, 2.3, S3.7, 2.4, and S2.8]). We only need to observe two facts:

(1) A function which is equal almost everywhere to a nearly upper semicontinuous function is itself nearly upper semicontinuous.

(2) The distance function from a subset of $\mathbf{R}$ is continuous.

For illustration, we sketch a fairly complicated proof of property (v), following the pattern of $\left[\mathbf{P}_{\mathbf{1}}\right.$, Theorem A7].

Choose an $\varepsilon>0$, and find a $\gamma \in(a, b)$ so that $\left|f_{a}^{c} f-I\right|<\varepsilon / 3$ for each $c \in[\gamma, b)$, and $|f(b)|(b-\gamma)<\varepsilon / 3$. Select a strictly increasing sequence $\left\{c_{n}\right\}_{n=0}^{\infty}$ in $[a, b)$ with $c_{0}=a$ and $\lim c_{n}=b$. By (iii), for each $n=1,2, \ldots$, there is a nearly upper semicontinuous $\delta_{n}:\left[c_{n-1}, c_{n}\right] \rightarrow \mathbf{R}_{+}$such that

$$
\sum_{i=1}^{p}\left|f\left(x_{i}\right)\right| A_{i}\left|-\int_{A_{i}} f\right|<\frac{\varepsilon}{3} 2^{-n}
$$

whenever $\left\{\left(A_{1}, x_{1}\right), \ldots,\left(A_{p}, x_{p}\right)\right\}$ is a $\delta_{n}$-fine subpartition of $\left[c_{n-1}, c_{n}\right]$. In view of observations (1) and (2), we may assume that

$$
\delta_{n}(x) \leq \min \left(\left|x-c_{n-1}\right|,\left|x-c_{n}\right|\right)
$$

for each $x \in\left(c_{n-1}, c_{n}\right), \delta_{1}\left(c_{0}\right) \leq c_{1}-c_{0}$, and

$$
\delta_{n}\left(c_{n}\right)=\delta_{n+1}\left(c_{n}\right) \leq \min \left(c_{n}-c_{n-1}, c_{n+1}-c_{n}\right), \quad n=1,2, \ldots
$$

Clearly, the function $\delta$ on $A$ defined by

$$
\delta(x)= \begin{cases}\delta_{n}(x) & \text { if } x \in\left[c_{n-1}, c_{n}\right], n=1,2, \ldots, \\ b-\gamma & \text { if } x=b\end{cases}
$$

is positive and nearly upper semicontinuous. We show that it belongs to $\Delta(f, A ; \varepsilon)$.

Let $P=\left\{\left(A_{1}, x_{1}\right), \ldots,\left(A_{p}, x_{p}\right)\right\}$ be a $\delta$-fine partition of $A$. After a suitable reordering, we may assume that $A_{i}=\left[t_{i-1}, t_{i}\right], i=1, \ldots, p$, where $a=t_{0}<$ $\cdots<t_{p}=b$. Replacing $\left(A_{i}, x_{i}\right)$ in $P$ by $\left\{\left(\left[t_{i-1}, x_{i}\right], x_{i}\right),\left(\left[x_{i}, t_{i}\right], x_{i}\right)\right\}$, whenever $x_{i} \in\left(t_{i-1}, t_{i}\right)$, we obtain a $\delta$-fine partition $Q$ of $A$ with $\sigma(f, Q)=\sigma(f, P)$. Thus with no loss of generality we may also assume that $x_{i}=t_{i-1}$ or $x_{i}=t_{i}$ for each $i=1, \ldots, p$. From this and the choice of $\delta$, we make the following conclusion: if

$$
P_{n}=\left\{\left(A_{i}, x_{i}\right) \in P: A_{i} \subset\left[c_{n-1}, c_{n}\right]\right\}
$$

for $n=1,2, \ldots$, and if $N$ is the first positive integer with $c_{N} \geq t_{p-1}$, then conditions (a) - (c) below are satisfied.

(a) $P_{n}$ is a $\delta_{n}$-fine partition of $\left[c_{n-1}, c_{n}\right]$ for $n=1, \ldots, N-1$.

(b) $P_{N}$ is a $\delta_{N}$-fine partition of $\left[c_{N-1}, t_{p-1}\right]$; in particular, $P_{N}$ is a $\delta_{N}$-fine subpartition of $\left[c_{N-1}, c_{N}\right]$. 
(c) $P=\left(\bigcup_{n=1}^{N} P_{n}\right) \cup\left\{\left(\left[t_{p-1}, b\right], b\right)\right\}$.

Now we have

$$
\begin{gathered}
|\sigma(f, P)-I| \leq\left|\sigma(f, P)-\int_{a}^{t_{p-1}} f\right|+\left|\int_{a}^{t_{p-1}} f-I\right|<\sum_{n=1}^{N-1}\left|\sigma\left(f, P_{n}\right)-\int_{c_{n-1}}^{c_{n}} f\right| \\
+\left|\sigma\left(f, P_{N}\right)-\int_{c_{N-1}}^{t_{p-1}} f\right|+|f(b)|\left(b-t_{p-1}\right)+\frac{\varepsilon}{3}<\frac{\varepsilon}{3} \sum_{n=1}^{N} 2^{-n}+2 \cdot \frac{\varepsilon}{3}<\varepsilon
\end{gathered}
$$

and the proof is completed.

8. LEMMA. Let $f$ be a function on an interval $A$, and let $\left\{B_{n}: n=1,2, \ldots\right\}$ be a disjoint family of subintervals of $A$ such that $f \in \mathscr{R}_{*}\left(B_{n}\right)$ for $n=1,2, \ldots$, and $f \in \mathscr{L}\left(A-\bigcup_{n \geq 1} B_{n}\right)$. Further let $W_{n}=\sup \left|\int_{C} f\right|$ where the supremum is taken over all intervals $C \subset B_{n}$, and suppose that $\sum_{n \geq 1} W_{n}<+\infty$. Then $f \in \mathscr{R}_{*}(A)$.

PROOF. Let $S=A-\bigcup_{n \geq 1} B_{n}$ and $I=(L) \int_{S} f+\sum_{n \geq 1} \int_{B_{n}} f$. Since $\left|\int_{B_{n}} f\right| \leq$ $W_{n}$, we see that $I$ is a well-defined real number. Choose an $\varepsilon>0$, and find an integer $N \geq 1$ with $\sum_{n>N} W_{n}<\varepsilon / 6$. Let $G=\bigcup_{n>N} \operatorname{int}\left(B_{n}\right), T=A-\left(G \cup \bigcup_{n=1}^{N} B_{n}\right)$, and let $\varphi, \psi$, and $h$ be, respectively, the functions $f \uparrow \bigcup_{n=1}^{N} B_{n}, f \uparrow T$, and $f \uparrow G$ extended to $A$ by zero. Thus $f=\varphi+\psi+h$, and it follows from Proposition 4 and Lemma 7 (iv) that $\varphi \in \mathscr{R}_{*}(A)$ and $\int_{A} \varphi=\sum_{n=1}^{N} \int_{B_{n}} f$. Since $T$ differs from $S$ only by a countable set, Proposition 4 implies that $\psi \in \mathscr{R}_{*}(A)$, and we have $\int_{A} \psi=(L) \int_{S} f$. Hence by Lemma 7, (i), the function $g=\varphi+\psi$ belongs to $\mathscr{R}_{*}(A)$. Consequently, we can find a nearly upper semicontinuous function $\delta_{g}: A \rightarrow \mathbf{R}_{+}$so that

$$
\left|\sigma(g, P)-\left[(L) \int_{S} f+\sum_{n=1}^{N} \int_{B_{n}} f\right]\right|<\frac{\varepsilon}{3}
$$

for each $\delta_{g}$-fine partition $P$ of $A$. By Lemma 7, (iii), there is a nearly upper semicontinuous function $\delta_{n}: B_{n} \rightarrow(0,1]$ such that

$$
\sum_{i=1}^{q}\left|f\left(z_{i}\right)\right| E_{i}\left|-\int_{E_{i}} f\right|<\frac{\varepsilon}{3} 2^{-n}
$$

for each $\delta_{n}$-fine subpartition $\left\{\left(E_{1}, z_{1}\right), \ldots,\left(E_{q}, z_{q}\right)\right\}$ of $B_{n}, n=1,2, \ldots$ In view of observation (2) in the proof of Lemma 7, we may assume that $\left(x-\delta_{n}(x)\right.$, $\left.x+\delta_{n}(x)\right) \subset B_{n}$ whenever $x \in \operatorname{int}\left(B_{n}\right)$. We define a nearly upper semicontinuous function $\delta_{h}: A \rightarrow(0,1]$ by setting

$$
\delta_{h}(x)= \begin{cases}\delta_{n}(x) & \text { if } x \in B_{n} \text { and } n>N, \\ 1 & \text { otherwise, }\end{cases}
$$

and we show that $\delta=\min \left(\delta_{g}, \delta_{h}\right)$ belongs to $\Delta(f, A ; \varepsilon)$.

To this end, let $P=\left\{\left(A_{1}, x_{1}\right), \ldots,\left(A_{p}, x_{p}\right)\right\}$ be a $\delta$-fine partition of $A$. For $n=$ $1,2, \ldots$, denote by $K_{n}$ the set of all the integers $k$ with $1 \leq k \leq p$ and $x_{k} \in \operatorname{int}\left(B_{n}\right)$, and set $P_{n}=\left\{\left(A_{k}, x_{k}\right) \in P: k \in K_{n}\right\}$ and $C_{n}=\operatorname{cl}\left(B_{n}-\bigcup_{k \in K_{n}} A_{k}\right)$. Then each $C_{n}$ is a union of at most two intervals, and by our choice of $\delta_{h}$, for each $n>N$, the collection $P_{n}$ is a $\delta_{n}$-fine subpartition of $B_{n}$; in particular, $B_{n}=C_{n} \cup \bigcup_{k \in K_{n}} A_{k}$. 
As $h=f$ on $G$ and $h=0$ on $A-G$, we have

$$
\begin{aligned}
|\sigma(f, P)-I| & \leq\left|\sigma(g, P)-\left[(L) \int_{S} f+\sum_{n=1}^{N} \int_{B_{n}} f\right]\right|+\left|\sigma(h, P)-\sum_{n>N} \int_{B_{n}} f\right| \\
& <\frac{\varepsilon}{3}+\left|\sum_{n \geq N} \sum_{k \in K_{n}} f\left(x_{k}\right)\right| A_{k}\left|-\sum_{n \geq N}\left[\sum_{k \in K_{n}} \int_{A_{k}} f+\int_{C_{n}} f\right]\right| \\
& \leq \frac{\varepsilon}{3}+\sum_{n \geq N} \sum_{k \in K_{n}}\left|f\left(x_{k}\right)\right| A_{k}\left|-\int_{A_{k}} f\right|+\sum_{n>N}\left|\int_{C_{n}} f\right| \\
& <\frac{\varepsilon}{3}+\frac{\varepsilon}{3} \sum_{n>N} 2^{-n}+2 \sum_{n>N} W_{n}<\varepsilon
\end{aligned}
$$

and the lemma is proved.

9. THEOREM. If $A$ is an interval, then $\mathscr{R}_{*}(A)=\mathscr{R}(A)$.

PROOF. In view of Theorem 2, it suffices to show that $\mathscr{D}(A) \subset \mathscr{R}_{*}(A)$. However, by means of Proposition 4 and Lemmas 7 and 8, this follows readily from the constructive definition of the Denjoy-Perron integral (see [S, Chapter VIII, §5] or [N, Chapter XVI, $\S \S 6$ and 7]).

10. REMARK. The proof of Theorem 9 does not generalize to higher dimensions (cf. Remark 6). Indeed, the proof is based on the possibility of obtaining the Henstock-Kurzweil integral by the Denjoy transfinite process, for which no satisfactory analogue in higher dimensions is known. Thus it is an open question whether Theorem 9 holds for the higher dimensional Henstock-Kurzweil integral (see [Ml]), or for its generalizations defined in $\left[\mathbf{M}, \mathbf{J K S}\right.$, and $\left.\mathbf{P}_{\mathbf{3}}\right]$.

\section{REFERENCES}

[AP] S. I. Ahmed and W. F. Pfeffer, A Riemann integral in a locally compact Hausdorff space, J. Austral. Math. Soc. Ser. A 41 (1986), 115-137.

[FM] J. Foran and S. Meinershagen, Some answers to a question of P. Bullen, Real Anal. Exchange 13 (1987-88), 256-277.

[H] R. Henstock, Theory of integration, Butterworths, London, 1963.

[JKS] J. Jarnik, J. Kurzweil and S. Schwabik, On Mawhin's approach to multiple nonabsolutely convergent integral, Casopis Pěst. Mat. 108 (1983), 356-380.

[K] J. Kurzweil, Nichtabsolut konvergente Integrale, Teubner Verlag, Leipzig, 1980.

[M] J. Mawhin, Generalized multiple Perron integrals and the Green-Goursat theorem for differentiable vector fields, Czechoslovak Math. J. 31 (1981), 614-632.

[Ml] R. M. McLeod, The generalized Riemann integral, Carus Math. Monos., 20, MAA, Washington, D.C., 1980.

[Ms] E. J. McShane, A unified theory of integration, Amer. Math. Monthly 80 (1973), 349-359.

[N] I. P. Natanson, Theory of functions of a real variable, vol. II, Ungar, New York, 1967.

[P1] W. F. Pfeffer, The Riemann-Stieltjes approach to integration, TWISK 187, NRIMS:CSIR, Pretoria, 1980.

$\left[\mathbf{P}_{\mathbf{2}}\right] \ldots$, The generalized Riemann integral in higher dimensions, Measure Theory and its Applications, Lecture Notes in Math., vol. 1033, Springer-Verlag, New York, 1983, pp. 269-275.

[P3] _ The divergence theorem, Trans. Amer. Math. Soc. 95 (1986), 665-685.

[Q] Queries, Real Anal. Exchange 12 (1986-87), 393.

[S] S. Saks, Theory of the integral, Dover, New York, 1964.

Department of Mathematics, University of California, Davis, California 95616 